\title{
Aplicabilidade da classificação de alcoolismo tipo A/tipo B
}

\author{
Aplicability of the type A/type B classification of alcoholics
}

Mário Sérgio Ribeiro', Luiz Cláudio Ribeiro², Grazielle Fialho de Souza³, Marcondes Garcia Antunes³, Rachel Bueno Nogueira ${ }^{3}$, Karolina Danielle Carvalho de Sousa ${ }^{3}$

\section{RESUMO}

Objetivos: Avaliar a aplicabilidade da tipologia e caracterizar os subtipos identificados. Métodos: Características de 300 homens alcoolistas atendidos em um programa ambulatorial foram submetidas à análise de cluster para separá-los em dois subgrupos de acordo com a tipologia de Babor et al. Efetivaram-se cruzamentos de dados (significância avaliada pelo Teste do qui-quadrado de Pearson) para se verificar a associação dos clusters com variáveis clínicas e demográficas. Resultados: Comparado ao outro grupo e pacientes, um dos clusters identificados foi caracterizado por um perfil de maior gravidade clínica. Pacientes do subtipo menos grave foram mais frequentemente $(65,3 \%)$ encaminhados a tratamentos simbólicos, enquanto pacientes do subtipo mais grave foram atendidos predominantemente $(58,5 \%)$ por abordagem exclusivamente farmacológica e aderiram mais ao tratamento proposto. Conclusões: Como os resultados identificaram subtipos de alcoolistas com distintas características, este estudo evidenciou a aplicabilidade clínica da tipologia de Babor et al. em nosso meio sociocultural'. Também aponta para a relevância de estudos tipológicos que possam contribuir para uma mais ampla compreensão dos aspectos etiológicos, preventivos e terapêuticos do alcoolismo.

\section{ABSTRACT}

Objective: To test the applicability of this typology and to characterize the identified subtypes. Methods: Characteristics of 300 alcoholic men attending an out-treatment program were submitted to cluster analysis for identification of two subgroups (clusters), according to the original classification. Cross-tabulations were then performed to test for possible association of identified clusters to demographic and clinical features. Statistical significance was given by Pearson chisquare tests. Results: Compared to the other group, one of the identified clusters was characterized by a more severe clinical profile. Patients of the mild subtype were principally $(65,3 \%)$ referred to symbolic treatments while patients of the severe subtype were mainly assisted (58,5\%) exclusively through a pharmacological approach and remained in treatment for longer periods of time. Conclusions: As the results identified subtypes of alcoholics with differential features, this study brought forth the clinical applicability of Babor et al. typology in our social-cultural context? Results also point to the relevance of typological studies that may contribute to a more adequate understanding of etiological, preventive and therapeutic aspects of alcoholism.

\section{Alcoholism, typology, cluster analysis, psychiatry.}

Keywords

1 Universidade Federal de Juiz de Fora (UFJF), Faculdade de Medicina, Departamento de Clínica Médica. Laboratório de Pesquisas em Personalidade, Álcool e Drogas (Lappda-UFJF).

2 Universidade Federal de Juiz de Fora (UFJF), Departamento de Estatística. Laboratório de Pesquisas em Personalidade, Álcool e Drogas (Lappda-UFJF). 3 Universidade Federal de Juiz de Fora (UFJF), Laboratório de Pesquisas em Personalidade, Álcool e Drogas (Lappda-UFJF). 


\section{INTRODUÇÃO}

No campo da clínica médica, as classificações tipológicas contribuem principalmente para otimizar o diagnóstico, sugerir o prognóstico, medidas preventivas e/ou direcionar o tratamento por meio de um simples esquema de classificação. As principais tipologias de alcoolismo desenvolvidas ao longo do século XX - Knight; Menninger; Jellinek; Conley e Prioleau; Von Knorring; Morey e Skinner; Zucker; Cloninger; Babor; Mezzich et al.; Johnson e Pickens - indicam a presença de dois tipos básicos de alcoolistas, mais frequentemente encontrados e descritos pelos autores. Um tipo seria caracterizado por início tardio, evolução mais lenta, menos complicações, menos comorbidade psicopatológica e melhor prognóstico. O outro tipo apresentaria predisposição genética, início precoce, evolução mais rápida, mais comorbidade psicopatológica, sintomas mais severos e pior prognóstico'.

No entanto, estudos mais recentes sobre subtipos de alcoolistas sugerem que ainda não há consenso acerca da natureza e do número de subtipos que podem ser utilizados para caracterizar a heterogeneidade clínica e etiológica presente nesses grupos de pessoas². Uma correta compreensão das limitações de cada tipologia, até o momento constituída, deve contribuir para a construção de outras classificações mais adequadas às necessidades dos pacientes e dos profissionais aos quais eles são encaminhados em busca de cuidados clínicos.

A classificação tipo A/tipo B de Babor et al. ${ }^{3}$ está entre as principais tipologias de alcoolismo e tem sido avaliada por diversos pesquisadores que, todavia, encontraram diferentes resultados em termos de validades de construto e preditiva dessa classificação $0^{4-12}$.

Babor et al. ${ }^{3}$ basearam sua tipologia na suposição de que a heterogeneidade entre os alcoolistas seria atribuída a uma complexa interação entre fatores genéticos, biológicos, psicológicos e socioculturais. Após revisarem a literatura acerca das tipologias de alcoolismo, a partir da metade do século XIX, os autores buscaram identificar características definidoras que poderiam resultar na identificação mais precisa de subtipos de alcoolistas. Realizaram, então, uma avaliação prospectiva de 321 alcoolistas de ambos os sexos, que utilizou instrumentos de mensuração previamente validados, procurando selecionar medidas confiáveis de um amplo espectro de fatores clínicos e psicossociais teoricamente relevantes para a avaliação multidimensional dos alcoolistas. Os dados foram submetidos a métodos estatísticos de redução de dados, resultando em 17 variáveis representativas. Essas 17 características foram, ainda, agrupadas em quatro categorias:
1. Fatores de risco pré-mórbidos: alcoolismo familiar; desordens na infância; dimensão de caráter bipolar; início dos problemas ligados ao álcool.

2. Severidade da dependência e outros comportamentos associados: doses de álcool consumidas; consumo para alívio; síndrome de dependência; uso de benzodiazepínicos e outras drogas associado ao consumo de álcool.

3. Cronicidade e consequências do consumo: condições médicas associadas; consequências físicas e sociais; severidade da dependência; anos de consumo pesado (cronicidade).

4. Comorbidade psicopatológica: sintomas depressivos; personalidade antissocial; ansiedade.

A seguir, os autores empregaram a análise de cluster e identificaram dois subtipos de alcoolistas, que claramente foram diferenciados com relação às 17 variáveis. Os alcoolistas do tipo A foram caracterizados como sendo mais conservadores - isto é, menos propensos a experiências e novidades - e seus problemas ligados ao álcool teriam início mais tardio. Também apresentariam: menor influência de fatores de risco relacionados à infância; menor grau de dependência; menores taxas de consequências físicas e sociais relacionadas ao consumo alcoólico; menores taxas de comorbidade psicopatológica e de alterações relacionadas ao trabalho e à família; progressão mais lenta do alcoolismo e melhor prognóstico. Já os alcoolistas do tipo B seriam mais experimentadores e mais tensos; apresentariam ainda: fatores de risco relacionados tanto a fatores familiares quanto à infância, início mais precoce dos problemas ligados ao álcool, maior grau de dependência, abuso de outras substâncias, consequências físicas e sociais mais sérias, maior frequência de comorbidade psicopatológica, progressão mais rápida do alcoolismo e pior prognóstico. Os dois subtipos foram avaliados prospectivamente aos 12 e 36 meses e também diferiram quanto ao tratamento ${ }^{3}$. Litt et al. ${ }^{4}$ e Brown et al. ${ }^{6}$, em estudos de replicação posteriores, também identificaram diferenças quanto à resposta de cada subtipo a diferentes modalidades terapêuticas.

Dando continuidade a uma linha de pesquisa que aborda a questão da tipologia de alcoolistas e discute a aplicabilidade das tipologias existentes em nosso meio ${ }^{13}$, os objetivos deste estudo foram: 1) avaliar a aplicabilidade da tipologia de Babor em pacientes de um programa de assistência a dependentes químicos (PADQ), empregando a análise de cluster (AC); 2) comparar as características destes dois clusters por procedimento de cruzamento de dados com variáveis sociodemográficas, clínicas e de personalidade; e 3) verificar a vinculação de cada um dos clusters às abordagens terapêuticas rotineiras do PADQ, bem como seus padrões de adesão ao tratamento proposto. 


\section{MÉTODO}

\section{Desenho do estudo}

Partiu-se de experimento natural, observacional, sem intervenção do grupo de pesquisadores na alocação dos sujeitos ou procedimentos terapêuticos. A rotina assistencial do PADQ já foi apresentada e discutida anteriormente ${ }^{13}$. Ressalta-se aqui que o autor principal participou como supervisor do trabalho clínico de residentes de psiquiatria envolvidos na assistência e que alguns dos demais autores acompanharam consultas médicas - gerais ou psiquiátricas - dos pacientes e/ou se responsabilizaram pela constituição dos bancos de dados informatizados.

O PADQ é um serviço público secundário, de referência para tratamento de dependentes de álcool e/ou outras substâncias psicoativas do Sistema Municipal de Saúde Mental de Juiz de Fora (SMSM-JF), município com mais de 500.000 habitantes, localizado na Zona da Mata mineira e com gestão plena do Sistema Único de Saúde. Trata-se de um programa multidisciplinar, com avaliações e intervenções realizadas por profissionais de distintas formações Enfermagem, Medicina (clínico geral e psiquiatras), Psicologia e Serviço Social. Os diagnósticos relativos ao alcoolismo e comorbidades psiquiátricas são realizados em base clínica e seguem os critérios da Classificação Internacional de Doenças, 10a revisão (CID-10).

O estudo foi autorizado pelo Comitê de Ética em Pesquisa - CEP/UFJF (parecer 072/2007).

\section{Sujeitos}

A totalidade de adultos, que tiveram seu primeiro atendimento no PADQ, no período compreendido entre outubro de 1997 e dezembro de 2004, foi de 727, pacientes com transtornos mentais e/ou do comportamento, associados ao uso de substâncias psicoativas. Destes, 157 tiveram diagnósticos relativos ao uso de álcool e outras substâncias psicoativas e 570 de transtorno mental e de comportamento decorrente, exclusivamente, do uso de álcool. A dependência de nicotina não foi considerada, nem como critério de inclusão nem de exclusão. Foram retirados das análises sete pacientes que se enquadravam aos critérios de exclusão do próprio programa assistencial - em especial, Uso Nocivo (F10.1), Síndrome Amnéstica (F10.6), Demência (F10.73) e outros comprometimentos cognitivos persistentes, decorrentes do uso de álcool (F10.74). Foram também excluídos do estudo: a) sujeitos que não completaram a avaliação inicial no PADQ (368); b) mulheres, tanto por seu número limitado (37 pacientes) para as análises estatísticas quanto pelo fato de terem sido avaliadas separadamente nos estudos de replicação da tipologia de Babor et al. (1992); c) sujeitos cujos prontuários informatizados não continham informações necessárias e suficientes para a análise tipológica, isto é, com elevado percentual (50\% ou mais) das variáveis estudadas sem resposta indicada. No total, foram excluídos desse estudo 420 casos e o grupo estudado ficou composto por 300 sujeitos: 226 dependentes exclusivamente de álcool e 74 com diagnósticos relativos ao uso de álcool e outras substâncias psicoativas (Figura 1).

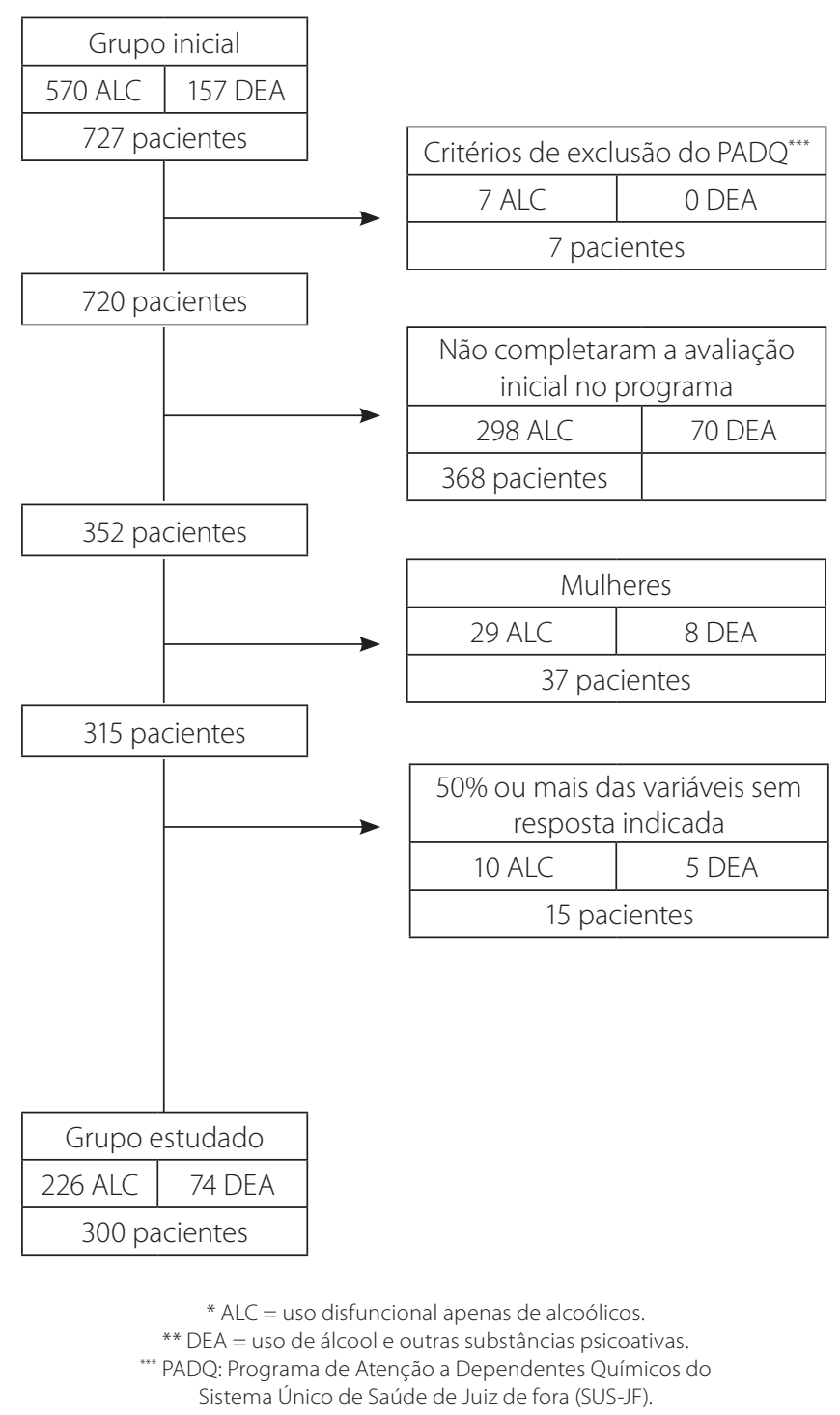

Figura 1. Procedimentos de seleção dos pacientes estudados.

\section{Identificação e adequação das variáveis}

Da mesma forma que outros estudos ${ }^{4-12}$, que tentaram replicar a tipologia tipo A/tipo B, também se procurou, neste estudo, utilizar variáveis o mais semelhantes possível das originais de Babor et al. ${ }^{3}$. Como os dados provenientes das avaliações realizadas no PADQ não permitem a extração de todas as medidas de forma idêntica às utilizadas no estudo original, fez-se necessário que algumas das variáveis disponíveis fossem adaptadas para que se assemelhassem - por 
adequação ou aproximação conceitual - àquelas utilizadas para a composição da tipologia aqui estudada.

Das 17 características utilizadas para a construção da tipologia tipo A/tipo B, foi possível identificar 15 variáveis idênticas, ou que se assemelhavam, em termos de conteúdo, às variáveis do estudo original³. Algumas delas foram extraídas diretamente das centenas de particularidades clínicas habitualmente avaliadas no PADQ e outras foram geradas por combinações de características semelhantes, com o objetivo de reduzir o número de dados sem resposta indicada ("missing") e obter as aqui denominadas "variáveis aproximadas". Não foi possível encontrar características que representassem as variáveis descritas como "Distúrbios da Infância" e "Severidade Vitalícia" (Quadro 1).

\section{Tratamento estatístico}

As respostas dos sujeitos às variáveis utilizadas foram então submetidas ao tratamento estatístico com uso do software SPSS for Windows 14.0. Para encontrar os dois subtipos, conforme proposto por Babor et al. ${ }^{3}$, foi empregada a técnica de AC pelo método K-means, opção pairwise. Esta técnica é empregada para tentar alocar os sujeitos da pesquisa em um número predeterminado de subgrupos relativamente homogêneos (clusters), utilizando-se um conjunto predeterminado de variáveis ${ }^{14}$. Para o procedimento de AC, as variáveis foram utilizadas com todas as categorias disponíveis.
Em seguida procedeu-se a um cruzamento de dados, comparando-se o padrão de respostas de cada um dos dois clusters identificados a cada uma das 15 variáveis utilizadas (Quadro 3).

Para a realização desses cruzamentos, as variáveis quantitativas - idade de início dos problemas com o álcool, anos de consumo pesado e escalas do Multifásico Minesota de Personalidade (MMPI) - foram transformadas em categóricas binárias. A fim de ressaltar as diferenças entre os dois clusters e melhor evidenciar o valor clínico da tipologia, as variáveis com a possibilidade de apresentação binária ou em três ou mais categorias foram avaliadas das duas formas. Para tanto, se utilizou: 1) no caso das variáveis quantitativas: de categorias "acima" ou "abaixo" da média do total de participantes; e também de categorias "acima" ou "abaixo" dos pontos de corte definidos pela literatura de referência; e 2) no caso das variáveis categóricas: da redução das categorias a duas possibilidades opostas, tais como "usar" ou "não usar" benzodiazepínicos, "usar" ou "não usar" outras drogas e "apresentar" ou "não apresentar" consequências físicas ou sociais do consumo. O teste do qui-quadrado de Pearson foi utilizado para se avaliar a significância das diferenças dos resultados encontrados para cada subtipo de alcoolistas com relação às categorias das variáveis consideradas ( $p<0,05)$; valores de $p$ compreendidos entre 0,1 e 0,05 foram considerados marginalmente significantes, sendo mantidos na análise dos dados em razão dos objetivos exploratórios desse estudo.

Quadro 1. Descrição e adequação das variáveis

\begin{tabular}{|c|c|}
\hline $\begin{array}{l}\text { Características dos subtipos descritos } \\
\text { por Babor et al. (1992) }\end{array}$ & Medidas usadas neste estudo \\
\hline 1. Alcoolismo familiar & História familiar de alcoolismo \\
\hline 2. Início dos problemas com o álcool & Média das idades de dois marcos: idade que passou a beber em maior quantidade e Idade em que o uso se tornou um problema \\
\hline 4. Consumo para alívio & $\begin{array}{l}\text { Resposta afirmativa a pelo menos um dos quatro itens: critério 2B do DSM-IV; critério ( da CID-10; beber para aliviar incômodo } \\
\text { (mal-estar) imediato; beber para aliviar incômodo (mal-estar) contínuo }\end{array}$ \\
\hline 3. Doses de álcool consumidas & Estimativa da quantidade média de álcool consumida por semana em período recente \\
\hline 5. Síndrome de dependência & $\begin{array}{l}\text { Variável criada pelo Lappda por recombinação de respostas ao DSM-IV e à CID-10 caracterizando síndrome de dependência como } \\
\text { predominantemente física ou restrita a fenômenos psicocomportamentais }\end{array}$ \\
\hline 6. Uso de benzodiazepínicos & $\begin{array}{l}\text { Escala de quatro itens abordando razões referidas para uso de medicações - "para parar de beber"; "para ansiedade/nervosis- } \\
\text { mo"; "para irritabilidade"; "para dormir" - no último mês }\end{array}$ \\
\hline 7. Uso de outras drogas & Presença ou não de síndrome de dependência a qualquer outra substância além do álcool (exceto tabaco) \\
\hline 8. Condições médicas & $\begin{array}{l}\text { Escala de dois itens: relato de ter apresentado problemas de saúde, internado ou feito tratamento médico no último ano; e } \\
\text { evidências de complicações clínicas pelo uso de álcool }\end{array}$ \\
\hline 9. Consequências físicas & Escala de 30 itens acerca de sinais/sintomas relatados nos últimos 30 dias \\
\hline 10. Consequências sociais & Escala de 15 itens de diferentes tipos de problemas (pessoais, institucionais, trabalhistas, agressão etc.) \\
\hline 11. Anos de consumo pesado & Idade à admissão no PADQ menos a idade em que o uso se tornou um problema \\
\hline 12. Dimensão de caráter bipolar & Escala de alcoolismo de MacAndrew do MMPI \\
\hline 13. Sintomas depressivos & Escala de D do MMPI \\
\hline 14. Sintomas de personalidade antissocial & Escala Pd do MMPI \\
\hline 15. Gravidade da ansiedade & "Taylor Manifest Anxiety Scale" do MMPI \\
\hline 16. Distúrbios da infância & Dados não disponíveis \\
\hline 17. Severidade vitalícia & Dados não disponíveis \\
\hline
\end{tabular}


Finalmente, foram verificados os padrões de adesão global e de utilização de diferentes modalidades assistenciais do PADQ por parte dos sujeitos pertencentes aos dois subgrupos (clusters) identificados, que também foram avaliados por meio do teste do qui-quadrado de Pearson.

\section{RESULTADOS}

Com a aplicação da AC, dos 300 sujeitos avaliados, 94 foram incluídos no cluster 1 e 206 no cluster 2 . De forma estatisticamente significante, o cluster 1 apresentou maior percentual de sujeitos com idade acima da média do total de participantes, menor taxa de católicos, menor percentual de sujeitos que trabalhavam no momento da avaliação e maior percentual dos que trabalhavam em sua profissão. De forma marginalmente significante, os pacientes do cluster 1 apresentaram maior percentual de sujeitos com tempo de união superior à média do grupo e com número de moradores em sua residência abaixo da média do grupo (Quadro 2).
O quadro 3 apresenta o percentual de respostas em cada categoria das variáveis estudadas em função da pertinência dos sujeitos a cada um dos dois clusters aqui identificados. Os resultados estatisticamente significantes indicam que, nos pacientes do cluster 1, o alcoolismo familiar foi mais frequente e os problemas com o consumo de alcoólicos foram percebidos em idade mais precoce. Esses pacientes fizeram mais uso de benzodiazepínicos, apresentaram consequências médicas mais frequentes e maior número de consequências físicas e sociais associadas ao etilismo; consumiram pesadamente por períodos mais prolongados de suas vidas; tiveram pontuações mais elevadas nas escalas do MMPI que avaliam aspectos ansiosos, depressivos e antissociais da personalidade; e foram encaminhados em maior proporção para tratamento exclusivamente farmacológico. Esses pacientes tiveram ainda maior pontuação na escala de MacAndrew ( $p=0,101$ no ponto de corte normativo) e também apresentaram tendência ao consumo disfuncional de outras substâncias psicoativas $(p=0,093)$. Por sua vez, os pacientes do cluster 2 apresentaram características opostas. As demais variáveis não apresentaram resultados ao menos marginalmente significantes.

Quadro 2. Percentuais de resposta dos sujeitos incluídos em cada cluster para variáveis sociodemográficas

\begin{tabular}{|c|c|c|c|c|c|c|}
\hline Variável & Categorias de resposta & $\begin{array}{c}\text { Cluster } 1 \\
(\mathrm{n}=94) \\
(\%)\end{array}$ & $\begin{array}{c}\text { Cluster } 2 \\
\text { ( } \mathrm{n}=206) \\
(\%)\end{array}$ & Total (\%) & $\begin{array}{l}\text { Sujeitos } \\
\text { (n) }\end{array}$ & $\begin{array}{l}\text { Valor } \\
\text { dep }\end{array}$ \\
\hline \multirow[t]{2}{*}{ Idade* } & Até 40 anos de idade & 41,5 & 54,4 & 50,3 & \multirow{2}{*}{300} & \multirow{2}{*}{0,038} \\
\hline & 41 anos de idade ou mais & 58,5 & 45,6 & 49,7 & & \\
\hline \multirow[t]{2}{*}{ Cor } & Branco & 69,7 & 71,3 & 70,8 & \multirow{2}{*}{291} & \multirow{2}{*}{0,77} \\
\hline & Não branco & 30,3 & 28,7 & 29,2 & & \\
\hline \multirow[t]{2}{*}{ Religião } & Católicos & 67,5 & 80,8 & 76,9 & \multirow{2}{*}{281} & \multirow{2}{*}{0,016} \\
\hline & Outras religiōes & 32,5 & 19,2 & 23,1 & & \\
\hline \multirow[t]{2}{*}{ Situação conjugal } & Estável & 47,3 & 50,0 & 49,2 & \multirow{2}{*}{297} & \multirow{2}{*}{0,667} \\
\hline & Instável ou sozinhos & 52,7 & 50,0 & 50,8 & & \\
\hline \multirow[t]{2}{*}{ Tempo de união* } & Até 13 anos & 41,2 & 54,8 & 50,9 & \multirow{2}{*}{175} & \multirow{2}{*}{0,100} \\
\hline & 14 anos ou mais & 58,8 & 45,2 & 49,1 & & \\
\hline \multirow[t]{2}{*}{ Número de moradores na residência* } & Até três moradores & 51,1 & 40,0 & 43,5 & \multirow{2}{*}{299} & \multirow{2}{*}{0,073} \\
\hline & Quatro moradores ou mais & 48,9 & 60,0 & 56,5 & & \\
\hline \multirow[t]{2}{*}{ Escolaridade } & Até primeiro grau & 77,7 & 76,1 & 76,6 & \multirow{2}{*}{299} & \multirow{2}{*}{0,767} \\
\hline & Maior que primeiro grau & 22,3 & 23,9 & 23,4 & & \\
\hline \multirow[t]{2}{*}{ Situação de trabalho } & Trabalha & 17,0 & 42,8 & 34,6 & \multirow{2}{*}{295} & \multirow{2}{*}{0,000} \\
\hline & Não trabalha & 83,0 & 57,2 & 65,4 & & \\
\hline \multirow[t]{2}{*}{ Trabalha na profissão } & Sim & 76,9 & 50,0 & 58,4 & \multirow{2}{*}{291} & \multirow{2}{*}{0,000} \\
\hline & Não & 23,1 & 50,0 & 41,6 & & \\
\hline \multirow[t]{2}{*}{ Dependência econômica de outros } & Não & 44,7 & 51,2 & 49,2 & \multirow{2}{*}{299} & \multirow{2}{*}{0,294} \\
\hline & Sim & 55,3 & 48,8 & 50,8 & & \\
\hline \multirow[t]{2}{*}{ Ser arrimo da família } & Não & 75,3 & 73,1 & 73,8 & \multirow{2}{*}{294} & 0699 \\
\hline & Sim & 24,7 & 26,9 & 26,2 & & 0,099 \\
\hline Renda pessoal & Menor que 1 salário mínimo & 52,3 & 43,6 & 46,2 & 297 & 0174 \\
\hline & Maior ou igual que 1 salário mínimo & 47,7 & 56,4 & 53,8 & 292 & 0,114 \\
\hline Renda familiar & Menor que 3 salários mínimos & 80,7 & 74,1 & 76,3 & 257 & $0>46$ \\
\hline & Igual ou maior que 3 salários mínimos & 19,3 & 25,9 & 23,7 & 231 & 0,240 \\
\hline
\end{tabular}

* Variáveis recodificadas em função da média do total de participantes. 
Quadro 3. Percentuais de resposta dos sujeitos incluídos em cada cluster para cada uma das demais variáveis estudadas

\begin{tabular}{|c|c|c|c|c|c|c|}
\hline Variável & Categorias de resposta & $\begin{array}{c}\text { Cluster } 1 \\
\text { (n= }=94) \\
(\%)\end{array}$ & $\begin{array}{c}\text { Cluster } 2 \\
\text { (n= 206) } \\
(\%)\end{array}$ & Total (\%) & $\begin{array}{c}\text { Sujeitos } \\
\text { (n) }\end{array}$ & $\begin{array}{l}\text { Valor } \\
\text { de } p\end{array}$ \\
\hline \multirow[t]{2}{*}{ Alcoolismo familiar } & Nenhuma ou não em parentes de $1^{\circ} \mathrm{grau}$ & 17,6 & 33,1 & 28,6 & \multirow{2}{*}{252} & \multirow{2}{*}{0,013} \\
\hline & Parentes de primeiro grau & 82,4 & 66,9 & 71,4 & & \\
\hline \multirow[t]{4}{*}{ Alcoolismo familiar } & Nenhuma ou não em parentes de $1^{\circ} \mathrm{grau}$ & 17,6 & 33,1 & 28,6 & \multirow{4}{*}{252} & \multirow{4}{*}{0,065} \\
\hline & Somente irmãos & 27,0 & 20,2 & 22,2 & & \\
\hline & Pai ou mãe & 52,7 & 42,1 & 45,2 & & \\
\hline & Pai e mãe & 2,7 & 4,5 & 4,0 & & \\
\hline \multirow[t]{2}{*}{ Início dos problemas com o álcool* } & Até 26 anos & 64,9 & 42,5 & 49,7 & \multirow{2}{*}{294} & \multirow{2}{*}{0,000} \\
\hline & 27 ou mais anos & 35,1 & 57,5 & 50,3 & & \\
\hline \multirow[t]{2}{*}{ Doses de álcool consumidas } & Até 126 doses por semana & 42,0 & 52,3 & 49,4 & \multirow{2}{*}{245} & \multirow{2}{*}{0,149} \\
\hline & 127 ou mais doses por semana & 58,0 & 47,7 & 50,6 & & \\
\hline \multirow[t]{2}{*}{ Consumo para alívio de sintomas } & Não & 19,8 & 19,9 & 19,9 & \multirow{2}{*}{287} & \multirow{2}{*}{0,979} \\
\hline & Sim & 80,2 & 80,1 & 80,1 & & \\
\hline \multirow[t]{3}{*}{ Síndrome de dependência } & Avaliação não explicitou diagnóstico & 13,8 & 10,2 & 11,3 & & \\
\hline & Sem diagnóstico de síndrome de dependência & 4,3 & 2,9 & 3,3 & 300 & 0,525 \\
\hline & Diagnóstico de síndrome de dependência & 81,9 & 86,9 & 85,3 & & \\
\hline $\begin{array}{l}\text { Síndrome de dependência - } \\
\text { Evidências de dependência física }\end{array}$ & $\begin{array}{l}\text { Não preencheu critérios suficientes para síndrome de } \\
\text { dependência }\end{array}$ & 4,9 & 3,2 & 3,8 & & \\
\hline & Não preencheu critérios para dependência física & 1,2 & 3,2 & 2,6 & 266 & 0,523 \\
\hline & Preencheu critérios para dependência física & 93,8 & 93,5 & 93,6 & & \\
\hline Uso de benzodiazepínicos & Não & 13,6 & 25,7 & 21,5 & 233 & 0037 \\
\hline & Sim & 86,4 & 74,3 & 78,5 & 250 & 0,002 \\
\hline Uso de benzodiazepínicos & Não & 13,6 & 25,7 & 21,5 & & \\
\hline & Uso por apenas um motivo & 56,8 & 55,9 & 56,2 & 233 & 0,037 \\
\hline & Uso por mais de um motivo & 29,6 & 18,4 & 22,3 & & \\
\hline Uso disfuncional de outras drogas & Não & 69,1 & 78,2 & 75,3 & 300 & $0 \cap 03$ \\
\hline & Sim & 30,9 & 21,8 & 24,7 & 300 & נקט, \\
\hline Uso de outras drogas & Não & 57,4 & 67,8 & 64,5 & & \\
\hline & Já usou, mas não de modo disfuncional & 11,7 & 10,2 & 10,7 & 299 & 0,196 \\
\hline & Usuário disfuncional à admissão & 30,9 & 22,0 & 24,7 & & \\
\hline Condições médicas - consequências e/ou & Não & 15,1 & 32,4 & 26,9 & 271 & 0 \\
\hline tratamento médico não psiquiátrico & Sim & 84,9 & 67,6 & 73,1 & 211 & 0,003 \\
\hline Condições médicas - consequências e/ou & Não apresenta consequências médicas & 15,1 & 32,4 & 26,9 & & \\
\hline tratamento médico não psiquiátrico & Consequência ou tratamento médico & 47,7 & 46,5 & 46,9 & 271 & 0,002 \\
\hline & Consequência e tratamento médico & 37,2 & 21,1 & 26,2 & & \\
\hline Consequências físicas & Não & 7,5 & 11,2 & 10,0 & 299 & 0,332 \\
\hline & Sim & 92,5 & 88,8 & 90,0 & 299 & 0,032 \\
\hline Consequências físicas: total de & Abaixo da média (Até 8 pontos) & 30,1 & 60,7 & 51,2 & 299 & 0,000 \\
\hline 30 itens* & Acima da média (a partir de 9 pontos) & 69,9 & 39,3 & 48,8 & 299 & 0,000 \\
\hline Consequências sociais - 15 itens* & Até 4 pontos & 49,5 & 62,6 & 58,6 & 297 & 0.034 \\
\hline & 5 ou mais pontos & 50,5 & 37,4 & 41,4 & 291 & 0,034 \\
\hline Anos de consumo pesado* & Até 7 anos & 18,4 & 66,8 & 51,3 & 271 & 0,000 \\
\hline & 8 ou mais anos & 81,6 & 33,2 & 48,7 & & \\
\hline Dimensão de caráter bipolar - & $\leq 24$ pontos & 23,9 & 34,9 & 31,8 & 242 & 0,101 \\
\hline Escala de MacAndrew do $\mathrm{MMPI}^{\ddagger}$ & $>24$ pontos & 76,1 & 65,1 & 68,2 & 242 & 0,101 \\
\hline Dimensão de caráter bipolar - & $\leq 26$ pontos & 40,3 & 49,1 & 46,7 & 242 & 0.217 \\
\hline Escala de MacAndrew do MMPI* & $>26$ pontos & 59,7 & 50,9 & 53,3 & & \\
\hline Sintomas depressivos - Escala D do MMPI & $\leq 70$ pontos & 20,9 & 85,1 & 67,7 & 248 & 0,000 \\
\hline & $>70$ pontos & 79,1 & 14,9 & 32,3 & 240 & \\
\hline Sintomas depressivos - Escala D do MMPI* & $\leq 63$ pontos & 7,5 & 70,7 & 53,6 & 248 & 0,000 \\
\hline & $>63$ pontos & 92,5 & 29,3 & 46,4 & 240 & 0,000 \\
\hline Sintomas de personalidade antissocial - & $\leq 70$ pontos & 25,4 & 92,3 & 74,2 & 248 & 0,000 \\
\hline Escala Pd do MMPI & $>70$ pontos & 74,6 & 7,7 & 25,8 & 240 & 0,000 \\
\hline Sintomas de personalidade antissocial - & $\leq 63$ pontos & 6,0 & 66,3 & 50,0 & 248 & 0000 \\
\hline Escala Pd do MMPI* & $>63$ pontos & 94,0 & 33,7 & 50,0 & 240 & 0,000 \\
\hline Gravidade da ansiedade - & $\leq 24$ pontos & 18,6 & 59,2 & 48,7 & 278 & ח \\
\hline Escala Taylor do MMPI* & $>24$ pontos & 81,4 & 40,8 & 51,3 & $\angle 28$ & 0,000 \\
\hline Adesão & Até 6 meses & 39,4 & 45,6 & 43,7 & 300 & 0310 \\
\hline & Maior que 6 meses & 60,6 & 54,4 & 56,3 & 300 & 0,310 \\
\hline Subprograma terapêutico utilizado no PADQ & Exclusivamente farmacológico & 58,5 & 34,7 & 42,2 & 258 & 0,000 \\
\hline & Simbólicos & 41,5 & 65,3 & 57,8 & 258 & 0,000 \\
\hline
\end{tabular}

\footnotetext{
* Variáveis recodificadas em função da média do total de participantes.
}

${ }^{\ddagger}$ Ponto de corte normativo ${ }^{14}$ 


\section{DISCUSSÃO}

\section{Aplicabilidade da tipologia}

Da mesma forma que no estudo original ${ }^{3}$ e nos principais estudos de replicação da tipologia ${ }^{4,6}$, foi possível a identificação de dois subgrupos de alcoolistas com características distintas. Os resultados indicaram que o cluster 1, aqui identificado, apresenta características clínicas que podem ser consideradas como mais graves do que aquelas identificadas para o cluster 2: portanto, os dois clusters serão, de agora em diante, designados como "tipo $+G$ " (mais grave) e "tipo -G" (menos grave). De forma semelhante à do estudo original, alcoolistas de menor gravidade - aqui denominados tipo -G (cluster 2), correspondentes ao tipo A de Babor et al. - foram identificados em maior proporção que os alcoolistas do tipo $+G$ (cluster 1), considerados mais graves, como os do tipo B de Babor et al.

Assim sendo, os resultados permitem considerar que a tipologia de Babor et al. possui aplicabilidade clínica em nosso meio.

\section{Caracterização dos subtipos}

Algumas das variáveis avaliadas pelos cruzamentos dos dados não evidenciaram seu valor propedêutico e prognóstico pleno, quando mantidas em um único formato; por essa razão, foram utilizadas em suas versões binárias e/ou com múltiplas categorias, o que permitiu melhor explicitar as diferenças clínicas entre os subtipos. Assim sendo, percebe-se que a apresentação binária da variável "Uso de benzodiazepínicos" revelou maior percentual de consumo pelos sujeitos do subtipo $+\mathrm{G}(86,4 \%)$ do que pelos do subtipo - $\mathrm{G}(74,3 \%)$, mesmo que se tenha registrado elevado consumo desses fármacos por ambos grupos. Todavia, observa-se melhor discriminação clínica entre os dois grupos, quando se avaliam possíveis motivações para seu consumo. Pacientes do subtipo $+G$ se diferenciam daqueles do subtipo - G em especial pelo uso de benzodiazepínicos por mais de um motivo.

Para a variável "alcoolismo familiar", embora a significância estatística tenha sido observada apenas quando da recodificação binária - com história familiar positiva presente em elevada proporção nos sujeitos dos dois subtipos, mas significativamente mais presente no subtipo $+G(82,4 \%$, $p=0,013)$-, aspectos clínicos relevantes também foram observados com a recodificação em quatro categorias. Assim sendo, as diferenças entre os dois tipos parecem ser cada vez mais evidentes com o crescimento do peso da carga genética - à exceção da categoria "pai e mãe", que, todavia, teve um número reduzido de representantes.

Em seu estudo de avaliação da tipologia de Babor et al. ${ }^{3}$, Schuckit et al. ${ }^{8}$ não evidenciaram que a história familiar tivesse contribuído significativamente para a diferenciação entre os subtipos A e B do grupo de pacientes por eles estu- dados. Os pesquisadores ressaltaram que a alta carga familiar dos indivíduos envolvidos em seu estudo provavelmente teria minimizado a capacidade de algumas medidas de intensidade da história familiar para diferenciar os grupos. A rigor, pode-se dizer que vieses de seleção dessa natureza possam ter influenciado os resultados do presente estudo, uma vez que $71,4 \%$ dos pacientes apresentavam história familiar positiva para alcoolismo - ainda que a história familiar tenha sido significativamente mais frequente no tipo $+G$ $(p=0,013)$. Por sua vez, Johnson e Pickens ${ }^{15}$ avaliaram uma tipologia ternária por eles desenvolvida - subtipos de alcoolistas caracterizados como brando, severo e antissocial com relação à predisposição familiar. Dentre os homens registrou-se, para o subtipo severo, uma densidade familiar de alcoolistas 3,5 vezes maior que a encontrada para um grupo de não alcoolistas (controles) e cerca de 2 vezes maior que a dos sujeitos do subtipo brando. Para os sujeitos do subtipo antissocial, a densidade familiar de alcoolismo ficou entre os níveis relatados pelos subtipos brando e severo - cerca de 2,6 vezes maior que os dos controles. Segundo os autores, os resultados evidenciariam a influência da carga familiar na gravidade do alcoolismo.

Quanto ao uso de outras drogas e à evidência de condições médicas não psiquiátricas, relacionadas ao consumo e etílicos, os resultados reforçam o raciocínio acima exposto para as variáveis "uso de benzodiazepínicos" e "alcoolismo familiar": a utilização de mais categorias pode revelar meIhor as diferenças clínicas entre os dois subgrupos. O uso disfuncional de outros psicoativos e a evidência de "consequências e tratamento médico" foram as categorias que discriminaram melhor os dois subtipos.

O consumo disfuncional de outras substâncias psicoativas é comumente relacionado ao subtipo que apresenta características clínicas mais graves ${ }^{2-4,69,15-18}$. Similarmente, este estudo também identificou que o subtipo $+G$ teria maior tendência ao uso disfuncional de outras substâncias psicoativas, da mesma forma que a maior probabilidade, já referida acima, de ter feito uso de benzodiazepínicos - um achado que deve ser levado em conta pelos profissionais responsáveis pela assistência médica a esses sujeitos.

Ainda que o mero registro de "consequências físicas" do consumo de alcoólicos não tenha se correlacionado a um dos clusters ( $p=0,332)$, a quantificação dessas consequências implicou a identificação de significância estatística $(p=0,000)$, com maior número de consequências associadas ao subtipo +G. Da mesma forma, as "consequências sociais" do uso de etílicos - qualitativamente registradas em $100 \%$ dos sujeitos estudados - também foram numericamente mais elevadas no subtipo $+G$, quando quantificadas $(p=0,034)$.

Os alcoolistas do subtipo $+G$ apresentaram pontuação mais elevada nas escalas do MMPI aqui avaliadas, sempre com elevada significância estatística $(p=0,000)$, à exceção 
da escala de alcoolismo de MacAndrew, cujos resultados apenas se aproximaram de significância marginal, com pontuações mais elevadas também no subtipo $+\mathrm{G}$. Ao se avaliar resultados observados em pesquisas anteriores ${ }^{19,20}$ nas quais se evidenciou que pontuações mais elevadas na escala de MacAndrew se refletiam nos resultados da escala $\mathrm{Pd}$, que avalia aspectos antissociais de personalidade - foi observado aqui uma tendência a tal associação $(p=0,156)$.

Penick et al.9, ao comparar 11 tipologias de alcoolismo, registraram que a maioria das tipologias descritas apresentava-se fortemente relacionada - em especial, quanto à idade de início do consumo, à presença de comorbidade psiquiátrica, ao sofrimento psicológico e à disfunção social na definição dos subtipos. Os autores observaram, ainda, que uma tipologia desenvolvida a partir das escalas do MMPI apresentou validade preditiva semelhante às quatro tipologias que obtiveram os melhores resultados. Os sujeitos aqui identificados como pertencentes ao subtipo $+G$ também foram associados a início mais precoce do consumo e dos problemas com o álcool; da mesma forma, tiveram maiores pontuações nas escalas do MMPI que avaliam características antissociais, depressivas e de ansiedade. O consumo pesado por período mais prolongado (variável "anos de consumo pesado") - significativamente mais elevado no subtipo $+G$ - reforça tal conclusão.

Para algumas das variáveis estudadas - "doses de álcool consumidas", "consumo para alívio" e "síndrome de dependência" - não foram observadas diferenças significativas entre os dois subtipos. Tais resultados indicam a gravidade dos pacientes aqui avaliados e apontam para o fato de que o simples diagnóstico "dependência" não é suficiente para evidenciar as diversas áreas de necessidades específicas de cada subgrupo de pacientes - de acordo com a perspectiva teórica de compreensão multidimensional do fenômeno da dependência a psicoativos.

\section{Vinculação de cada um dos clusters às abordagens terapêuticas e padrões de adesão}

A validade preditiva da tipologia de Babor pode ser avaliada, de forma prospectiva, verificando-se o comportamento de cada subtipo com relação a diferentes abordagens terapêuticas. Em estudos de seus autores ${ }^{3,21}$, os dois tipos mantiveram padrões diferenciáveis, quando avaliados aos 12 e 36 meses após tratamento. Em todas as comparações, os alcoolistas do tipo B apresentaram mais problemas com a bebida e maiores prejuízos relacionados ao álcool; aos 12 meses, 64\% dos identificados como pertencentes ao tipo B já haviam recaído e precisado de novo tratamento, ao passo que o mesmo ocorreu com apenas 45\% dos alcoolistas do tipo A (Babor et al., 1992; Babor et al., 1994).

Ainda que o desenho deste estudo - um experimento natural, sem intervenção dos pesquisadores na alocação dos sujeitos ou procedimentos terapêuticos - não permita avaliar a validade preditiva da tipologia, é possível, todavia, discutir o prognóstico dos subtipos por seu comportamento de adesão global e sua associação às abordagens terapêuticas utilizadas. Assim sendo, verificou-se que:

a) Apesar de não se ter evidenciado significância estatística $(p=0,310)$, é clinicamente relevante observar que o tipo $+\mathrm{G}$ obteve melhor percentual de adesão superior (maior que 6 meses) ao tratamento proposto;

b) De forma estatisticamente significante ( $p=0,000)$, os pacientes do tipo $+G$ foram atendidos em sua maioria $(58,5 \%)$ por abordagem exclusivamente farmacológica, ao passo que os pacientes do tipo - G foram encaminhados com mais frequência $(65,3 \%)$ a tratamentos simbólicos ${ }^{13}$ que, todavia, não excluem a utilização de psicofármacos ao longo do tratamento.

Tais resultados aparentemente paradoxais - melhor adesão do subtipo mais grave - sugerem que, conforme evidenciado em estudos anteriores ${ }^{22}$, o PADQ tem destinado a esses pacientes um tratamento adequado a suas necessidades mais amplas. A rigor, pacientes clínica e psiquiatricamente mais graves necessitam de fato de abordagem farmacológica, ao passo que sujeitos com quadros menos graves - possivelmente sem a presença de comorbidade relevante - podem ser atendidos em programas menos abrangentes e até por grupos de autoajuda ${ }^{23-26}$.

\section{Limitações}

Entre as limitações desse estudo ressalta-se o fato de: a) não ter sido possível a identificação de 2 das 17 dimensões utilizadas por Babor et al. ${ }^{3}$ - "distúrbios da infância" e "severidade vitalícia"; e b) ter sido feito uso de algumas variáveis não idênticas às do estudo original - uma dificuldade já referida por diversos autores ${ }^{7-10,27}$.

Como no estudo original, os dados aqui utilizados decorrem, em parte, de instrumentos de autorrelato, que podem ser respondidos de forma distorcida pelos sujeitos do estudo. As diferenças entre os participantes deste estudo e do estudo original - em especial quanto à composição sociodemográfica e a fatores culturais, conforme já apontados por Windle e Scheidt ${ }^{16}$ - poderiam limitar os resultados. Todavia, deve-se ressaltar que, ainda assim, os resultados encontrados foram consistentes com a classificação tipo A/tipo B.

Como em todos os experimentos naturais, existe uma limitada possibilidade de generalização dos resultados. Contudo, para além de sua implícita contribuição para a efetividade das práticas em saúde coletiva, ao avaliar uma "situação de vida real"28,29, os resultados aqui apresentados reforçam a viabilidade da utilização de instrumentos estatísticos aliados ao raciocínio clínico na abordagem de fenômenos tipológicos. 


\section{CONCLUSÃO}

Ao identificar subtipos de alcoolistas com distintas características clínicas, este estudo evidenciou a aplicabilidade clínica da tipologia de Babor et al. em nosso meio sociocultural. As relativas inconsistências acima discutidas, quanto a características mais precisas e/ou mais facilmente avaliáveis, em quaisquer contextos terapêuticos e/ou culturais, apontam para a relevância de estudos dessa natureza, de modo a superar a limitada utilidade cotidiana das tipologias já propostas. De qualquer forma, a aplicação de tipologias assim identificadas em desenhos experimentais pode, de fato, contribuir para uma compreensão mais adequada dos aspectos etiológicos, preventivos e terapêuticos do alcoolismo.

\section{REFERÊNCIAS}

1. Ribeiro MS, Guirro UBP, Baldi BG. Tipologias em alcoolismo: diagnóstico e terapêutica. Arq Bras Psiquiatr Neurol Med Legal. 2002;80/81:24-32.

2. Babor TF, Caetano R. Subtypes of substance dependence and abuse: implications for diagnostic classification and empirical research. Addiction, 2006;101(1):104-10.

3. Babor TF, Hofmann M, Del Boca FK, Hesselbrock V, Meyer RE, Dolinsky ZS, et al. Types of alcoholics, l: evidence for an empirically derived typology based on indicators of vulnerability and severity. Arch Gen Psychiatry. 1992;49:599-08.

4. Litt MD, Babor TF, DelBoca FK, Kadden RM, Cooney NL. Types of alcoholics, II: Applications of an empirically derived typology to treatment matching. Arch Gen Psychiatry. 1992:49:609-14.

5. Mezzich A, Tarter R, Kirisci L, Clark D, Buckstein 0, Martin C. Subtypes of early age onset alcoholism. Alcohol Clin Exp Res. 1993;17(4):767-70.

6. Brown J, Babor TF, Litt MD, Kranzler HR. The type A/type B distinction - Subtyping alcoholics according to indicators of vulnerability and severity. Ann NY Acad Sci. 1994;708:23-33.

7. Ball SA, Carroll KC, Babor TF, Rounsaville BJ. Subtypes of cocaine abusers: support for a type A/type B distinction. J Consult Clin Psychol. 1995;63:115-24.

8. Schuckit MA, Tipp JE, Smith TL, Shapiro E, Hesselbrock VM, Bucholz KK, et al. An evaluation of type A and type B alcoholics. Addiction. 1995;90(9):1189-03.

9. Penick EC, Nickel EJ, Powell BJ, Liskow BI, Campbell J, Dale TM, et al. The comparative validity of eleven alcoholism typologies. J Stud Alcohol. 1999;60(2):188-02.

10. Carpenter KM, Hasin DS. Reliability and discriminant validity of the type I/II and type A/B alcoholic subtype classifications in untreated problem drinkers: a test of the Apollonian Dionysian hypothesis. Drug Alcohol Depend. 2001;63:51-67.

11. Del Boca FK. Two subtypes or more, much work remains: a commentary on Windle \& Scheidt. Addiction. 2004;99:1609.

12. Driessen M, Lange W, Junghanns K, Wetterling T. Proposal of a comprehensive clinical typology of alcohol withdrawal - a cluster analysis approach. Alcohol Alcohol. 2005;40:308-13.
13. Ribeiro MS, Ribeiro LC, Souza GF, Garcia MA, Oliveira LN. Avaliação dos tipos 1 e 2 de alcoolismo de Cloninger em homens participantes de um programa de tratamento ambulatorial. Rev Psiq Clín. 2008;35(2):39-48.

14. Everitt BS, Dunn G (eds.). Applied multivariate data analysis. London: Edward Arnold; 1999.

15. Johnson E0, Pickens RW. Familial transmission of alcoholism among nonalcoholics and mild, severe, and dissocial subtypes of alcoholism. Alcohol Clin Exp Res. 2001;25:661-66.

16. Windle M, Scheidt DM. Alcoholic subtypes: are two sufficient? Addiction. 2004;99(12):1508-19.

17. Cardoso JM, Neves R, Barbosa A, Ismail F, Pombo S. Neter alcoholic typology. Alcohol Alcohol. 2006:41:133-9.

18. Hesselbrock VM, Hesselbrock MN. Are there empirically supported and clinically useful subtypes of alcohol dependence? Addiction. 2006;101(1):97-03.

19. MacAndrew C. What the MAC tells us about men alcoholics: An interpretative review. Quart J Stud Alc. 1981;42:604-25.

20. Wolf AW, Schubert DSP, Patterson M, Grande T, Pendleton L. The use of the MacAndrew Scale in detecting substance abuse and antisocial personality. J Pers Assess. 1990;54(3/4):747-55.

21. Babor TF, Hesselbrock V, Meyer RE, Shoemaker W. Introduction: method and theory in the classification of alcoholics. Ann NY Acad Sci. 1994;708:1-6.

22. Ribeiro MS, Alves MJM, Guirro UBP, Baldi BG. Alcoolismo: a influência do reconhecimento da co-morbidade na adesão de pacientes ao programa terapêutico. J Bras Psiq. 2004;53(2):124-32.

23. Moyer A, Finney JW, Swearingen CE, Vergun P. Brief interventions for alcohol problems: a meta-analytic review of controlled investigations in treatment-seeking and non-treatment-seeking populations. Addiction. 2002;97(3):279.

24. Tucker JA, Vuchinich RE, Rippens PD. Environmental contexts surrounding resolution of drinking problems among problem drinkers with different help-seeking experiences. J Stud Alcohol. 2002;63(3):334-41.

25. Gossop M, Harris J, Best D, Man LH, Manning V, Marshall J, et al. Is attendance at alcoholics anonymous meetings after inpatient treatment related to improved outcomes? A 6-month follow-up study. Alcohol Alcohol. 2003;38(5):421-26.

26. Moos RH, Moos BS. Long-term influence of duration and intensity of treatment on previously untreated individuals with alcohol use disorders. Addiction. 2003;98(3):325.

27 Epstein EE, Labouvie E, McCrady BS, Jensen NK, Hayaki J. A multi-site study of alcohol classification and overlap of unidimensional and multi-dimensional typologies. Addiction. 2002;97:1041-53.

28. Bao Y, Duan N, Fox SA. Is some provider advice on smoking cessation better than no advice? Health Serv Res. 2006;41(6):2114-35.

29. Haro JM, Suarez D, Novick D, Brown, Usall J, Naber D, et al. Three-year antipsychotic effectiveness in the outpatient care of schizophrenia: Observational versus randomized studies results. Eur Neuropsychopharmacol. 2006;17(4):235-44. 